PROCEEDINGS OF THE

AMERICAN MATHEMATICAL SOCIETY

Volume 139, Number 4, April 2011, Pages 1137-1140

S 0002-9939(2010)10523-3

Article electronically published on August 30, 2010

\title{
THEOREMS OF W. W. STOTHERS AND THE JACOBIAN CONJECTURE IN TWO VARIABLES
}

\author{
EDWARD FORMANEK
}

(Communicated by Ted Chinburg)

\begin{abstract}
Differential equations of the form $r p(z) q^{\prime}(z)-s p^{\prime}(z) q(z)=\gamma p(z)$ or $r p(z) q^{\prime}(z)-s p^{\prime}(z) q(z)=\gamma$, where $\gamma$ is a nonzero complex number and $r, s$ are positive integers, have arisen in attempts to solve the two-variable Jacobian Conjecture. Solutions of such equations, in which $p(z)$ and $q(z)$ are monic complex polynomials of positive degrees $r$ and $s$, give rise to extra-special pairs of polynomials in the sense of W. W. Stothers. Stothers showed that, modulo automorphisms of $\mathbb{C}[z]$, there are only finitely many extra-special pairs of a given degree $n$. This implies that, modulo automorphisms of $\mathbb{C}[z]$, there are only finitely many solutions of the above differential equations in which $p(z)$ and $q(z)$ are monic polynomials of given degrees $r$ and $s$.
\end{abstract}

\section{Introduction AND REVIEW OF Stothers' WORK}

The purpose of this paper is to point out a connection between certain differential equations which have arisen in attempts to establish the two-variable Jacobian Conjecture and the work of W. W. Stothers on the polynomial abc-conjecture.

In this first section, we review Stothers' work. In the next section, we prove our main result, Theorem 4. It follows from Stothers' theorems and says that, modulo a natural equivalence relation, certain differential equations have only finitely many solutions which are polynomials of given fixed degrees. The final section of the paper explains how polynomial solutions of such differential equations have arisen in attempts to prove the two-variable Jacobian Conjecture.

For a polynomial $f(z) \in \mathbb{C}[z]$ in one variable, let $d(f)$ be the degree of $f$ and let $c(f)$ be the number of distinct roots of $f$. Then $d(f) \geq c(f)$, with equality if and only if $f$ has no multiple roots. The polynomial abc-theorem is the following.

Theorem 1 (W. W. Stothers [4, Theorem 1.1]). Let $p(z), q(z) \in \mathbb{C}[z]$ be relatively prime monic polynomials of positive degree $n$. Then

$$
c(p)+c(q)+c(p-q) \geq n+1 .
$$

There have been many generalizations of this theorem, and there is a related abc-conjecture in number theory which would have many consequences (see [2]). Stothers investigated when the inequality in Theorem 1 becomes an equality, which led him to make the following definitions.

Received by the editors October 2, 2007 and, in revised form, April 28, 2009.

2010 Mathematics Subject Classification. Primary 14R15, 11C08.

Key words and phrases. Jacobian Conjecture, polynomial abc-theorem.

(C)2010 American Mathematical Society

Reverts to public domain 28 years from publication 
Definition. A pair $(p, q)$ of polynomials in $\mathbb{C}[z]$ is special of degree $n$ if $p$ and $q$ are relatively prime monic polynomials of degree $n$ and $c(p)+c(q)+c(p-q)=n+1$. The pair $(p, q)$ is extra-special of degree $n$ if it is special and $p-q$ has no multiple roots.

Definition. Let $(p, q),\left(p_{1}, q_{1}\right)$ be pairs of monic polynomials in $\mathbb{C}[z]$. Then $(p, q)$ and $\left(p_{1}, q_{1}\right)$ are $\infty$-equivalent if there are $\alpha \in \mathbb{C}^{*}, \beta \in \mathbb{C}$ such that $p_{1}(z)=$ $\alpha^{-d(p)} p(\alpha z+\beta), q_{1}(z)=\alpha^{-d(q)} q(\alpha z+\beta)$.

In other words, $\left(p_{1}, q_{1}\right)$ is obtained from $(p, q)$ by applying an automorphism of $\mathbb{C}[z]$ and then multiplying by the right constants to obtain monic polynomials. If $(p, q)$ is special (resp., extra-special) of degree $n$, so is an $\infty$-equivalent pair $\left(p_{1}, q_{1}\right)$.

His main theorem is that there is a one-to-one correspondence between certain subgroups of index $n$ in a free group of rank 2 and $\infty$-equivalence classes of special pairs of degree $n$. The fact that a free group of finite rank has only finitely many subgroups of index $n$ then yields the following theorem.

Theorem 2 (W. W. Stothers [4, Corollary 2.3]). For each positive integer n, there are only finitely many $\infty$-equivalence classes of special pairs of degree $n$.

Stothers' proofs of Theorems 1 and 2 depend on the theory of automorphic functions. There are elementary proofs of Theorem 1 (e.g., [2, p. 1225]), but not of Theorem 2.

\section{Polynomial solutions of SOME DifFEREnTial EQUATIONS}

We now apply Theorems 1 and 2 to polynomial solutions of certain differential equations.

Theorem 3. Let $p$ and $q$ be monic complex polynomials of positive degrees $r$ and $s$.

(a) Suppose that $r p q^{\prime}-s p^{\prime} q=\gamma p$ for some $\gamma \in \mathbb{C}^{*}$, and let $u$ be the monic greatest common divisor of $p^{s}$ and $q^{r}$. Then $\left(p^{s} / u, q^{r} / u\right)$ is an extra-special pair.

(b) Suppose that $r p q^{\prime}-s p^{\prime} q=\gamma$ for some $\gamma \in \mathbb{C}^{*}$. Then $\left(p^{s}, q^{r}\right)$ is an extraspecial pair.

Proof. (a) Since $u$ is the monic greatest common divisor of $p^{s}$ and $q^{r}, p^{s} / u$ and $q^{r} / u$ are relatively prime monic polynomials of the same positive degree. (The degree is positive, since if $d\left(p^{s} / u\right)=d\left(q^{r} / u\right)=0$, then $p^{s}=q^{r}$ and $r p q^{\prime}-s p^{\prime} q=0$.)

Set $h=p^{s}-q^{r}$, so that $d(h)<r s$, and let $\alpha \in \mathbb{C}^{*}$ be the coefficient of $z^{d(h)}$ in $h$. A computation shows that

$$
r h q^{\prime}-h^{\prime} q=\gamma p^{s}
$$

The highest degree term on the left side of (1) is $\alpha(r s-d(h)) z^{d(h)+s-1}$, so $d(h)+s-1=d\left(p^{s}\right)=r s$ and

$$
d(h)=r s-s+1
$$


The equation $r p q^{\prime}-s p^{\prime} q=\gamma p$ implies that every root of $p$ is a root of $q$ and that $q$ has no multiple roots. Hence we can write

$$
\begin{gathered}
p=\left(z-\alpha_{1}\right)^{a_{1}} \cdots\left(z-\alpha_{k}\right)^{a_{k}}\left(z-\beta_{1}\right)^{b_{1}} \cdots\left(z-\beta_{\ell}\right)^{b_{\ell}}, \\
r=d(p)=\sum a_{i}+\sum b_{j}, \\
q=\left(z-\alpha_{1}\right) \cdots\left(z-\alpha_{k}\right)\left(z-\beta_{1}\right) \cdots\left(z-\beta_{\ell}\right)\left(z-\gamma_{1}\right) \cdots\left(z-\gamma_{m}\right), \\
s=d(q)=k+\ell+m,
\end{gathered}
$$

where the notation is chosen so that $s a_{1}, \ldots, s a_{k} \geq r$ and $s b_{1}, \ldots, s b_{\ell}<r$. By hypothesis, $p$ and $q$ have positive degrees, but the notation is not meant to imply that all of $k, \ell, m$ are nonzero.

Dividing $r p q^{\prime}-s p^{\prime} q=\gamma p$ by $p q$ gives

$$
\sum\left(r-s a_{i}\right) /\left(z-\alpha_{i}\right)+\sum\left(r-s b_{j}\right) /\left(z-\beta_{j}\right)+\sum\left(r /\left(z-\gamma_{e}\right)\right)=\gamma / q
$$

which implies that $r \neq s a_{i}$ for all $i$, since otherwise $\alpha_{i}$ would be a pole of the right hand side of (2), but not of the left hand side.

The monic greatest common divisor of $p(z)^{s}$ and $q(z)^{r}$ is

$$
\begin{gathered}
u=\left(z-\alpha_{1}\right)^{r} \cdots\left(z-\alpha_{k}\right)^{r}\left(z-\beta_{1}\right)^{s b_{1}} \cdots\left(z-\beta_{\ell}\right)^{s b_{\ell}}, \text { so } \\
s \sum a_{i}-r k=d\left(p^{s} / u\right)=d\left(q^{r} / u\right)=r(\ell+m)-s \sum b_{j}, \text { and } \\
d\left(\left(p^{s}-q^{r}\right) / u\right)=d(h / u)=d\left(p^{s} / u\right)-s+1 .
\end{gathered}
$$

Furthermore, $c\left(p^{s} / u\right)=k, c\left(q^{r} / u\right)=\ell+m, s=k+\ell+m$, so

$$
\begin{aligned}
& \left.c\left(p^{s} / u\right)+c\left(q^{r} / u\right)+d\left(p^{s}-q^{r}\right) / u\right) \\
& \quad=k+\ell+m+d\left(p^{s} / u\right)-s+1=d\left(p^{s} / u\right)+1 .
\end{aligned}
$$

By Theorem 1,

$$
c\left(p^{s} / u\right)+c\left(q^{r} / u\right)+c\left(\left(p^{s}-q^{r}\right) / u\right) \geq d\left(p^{s} / u\right)+1 .
$$

Combining formulas (3) and (4) gives

$$
\begin{aligned}
& d\left(p^{s} / u\right)+1=c\left(p^{s} / u\right)+c\left(q^{r} / u\right)+d\left(\left(p^{s}-q^{r}\right) / u\right) \\
& \quad \geq c\left(p^{s} / u\right)+c\left(q^{r} / u\right)+c\left(\left(p^{s}-q^{r}\right) / u \geq d\left(p^{s} / u\right)+1,\right.
\end{aligned}
$$

so all inequalities become equalities and $d\left(\left(p^{s}-q^{r}\right) / u\right)=c\left(\left(p^{s}-q^{r}\right) / u\right)$. Thus $\left(p^{s}-q^{r}\right) / u$ has no multiple roots and $\left(p^{s} / u, q^{r} / u\right)$ is an extra-special pair.

(b) Note that the equation $r p q^{\prime}-s p^{\prime} q=\gamma$ implies that $p$ and $q$ are relatively prime, and set $t=p q$. Then

$$
r p t^{\prime}-(r+s) p^{\prime} t=\gamma p
$$

and the greatest common divisor $u$ of $p^{r+s}$ and $t^{r}$ is equal to $p^{r}$. By (a), we have that $\left(p^{r+s} / u, t^{r} / u\right)$ is an extra-special pair. But $p^{r+s} / u=p^{s}$ and $t^{r} / u=q^{r}$.

Theorem 4. Let $r, s$ be positive integers. Then there are only finitely many $\infty$ equivalence classes of pairs of monic polynomials $p, q \in \mathbb{C}[z]$ such that $p$ has degree $r, q$ has degree $s$, and $r p q^{\prime}-s p^{\prime} q$ is equal to either $\gamma p$ or $\gamma$ for some nonzero complex number $\gamma$.

Proof. The degree of $p^{s} / u$ is bounded above by $r s$. The map $(p, q) \rightarrow\left(p^{s} / u, q^{r} / u\right)$ preserves $\infty$-equivalence classes and induces a one-to-one map from $\infty$-equivalence classes of pairs $(p, q)$ satisfying the hypotheses of Theorem 4 to $\infty$-equivalence classes of extra-special pairs of degree $\leq r s$. By Theorem 2 this last set is finite. 
A pair of monic polynomials of degrees $r, s$ is defined by $r+s$ parameters (their coefficients), the differential equations of Theorem 4 impose $r+s-2$ conditions on these parameters, and an $\infty$-equivalence class of solutions is two-dimensional as an affine variety. Hence general principles suggest that the set of $\infty$-equivalence classes ought to be a zero-dimensional variety - i.e. finite - for given $r$ and $s$. Of course this is not a proof.

\section{Connection with the two-variable Jacobian Conjecture}

The Jacobian matrix of polynomials $f=f(x, y), g=g(x, y) \in \mathbb{C}[x, y]$ is the $2 \times 2$ matrix

$$
J(f, g)=\left[\begin{array}{ll}
\partial f / \partial x & \partial f / \partial y \\
\partial g / \partial x & \partial g / \partial y
\end{array}\right]
$$

If $\mathbb{C}[f, g]=\mathbb{C}[x, y]$, the chain rule implies that $J(f, g)$ is an invertible matrix. The Jacobian Conjecture in two variables is that the converse is true.

Jacobian Conjecture. If $J(f, g)$ is invertible, then $\mathbb{C}[f, g]=\mathbb{C}[x, y]$.

There is also an $n$-variable Jacobian Conjecture. There is strong evidence for the two-variable Jacobian Conjecture, but not for the $n$-variable conjecture. The one-variable Jacobian Conjecture is true and trivial.

The differential equations of Theorems 3 and 4 stem from the works of S. S. Abhyankar and T.-T. Moh on the two-variable Jacobian Conjecture, especially [1, 3. They appear explicitly in [3, Prop. A3, p. 205] and [3, Prop. A5, p. 207].

The way they appear is the following. After lengthy maneuvers which include changing variables and introducing fractional and negative fractional powers of $x$, one is led to equations which include

$$
\gamma x^{r a+s a-1}=\operatorname{det}\left(J\left(x^{r a} p(y), x^{s a} q(y)\right)\right)=\operatorname{det}\left[\begin{array}{ll}
\operatorname{rax}^{r a-1} p(y) & x^{r a} p^{\prime}(y) \\
\operatorname{sax}{ }^{s a-1} q(y) & x^{r a} q^{\prime}(y)
\end{array}\right],
$$

where $a$ is a rational number, $\gamma \in \mathbb{C}^{*}$, and $p(y), q(y)$ are polynomials in $y$ of positive degrees $r$ and $s$. Expanding the determinant and simplifying gives a differential equation $r p(y) q^{\prime}(y)-s p^{\prime}(y) q(y)=\gamma / a$ of the kind we studied.

\section{REFERENCES}

[1] S. S. Abhyankar, Some remarks on the Jacobian question, Notes by M. van der Put and W. Heinzer, updated by A. Sathaye, Proc. Indian Acad. Sci. (Math. Sci.) 104 (1994), 515-542. MR:1314394(95m:14013)

[2] A. Granville and T. J. Tucker, It's as easy as abc, Notices Amer. Math. Soc. 49 (2002), 1224-1231. MR1930670 (2003f:11044)

[3] T.-T. Moh, On the global Jacobian Conjecture and the configuration of roots, J. Reine Angew. Math. 340 (1983), 140-212. MR691964 (84m:14018)

[4] W. W. Stothers, Polynomial identities and Hauptmoduln, Quart. J. Math. Oxford 32 (1981), 349-370. MR625647 (83m:12006)

52 Living Edens Court, Las Vegas, Nevada 89148

E-mail address: formanek@math.psu.edu 